\title{
Matière première humaine
}

\section{Erhard Taverna}

Dr méd., membre de la rédaction

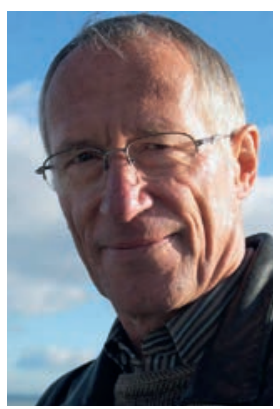

L'être humain, vivant ou mort, est utilisé depuis belle lurette comme réserve de pièces de rechange et pharmacie. Ceux qui reposent en paix laissent derrière eux une enveloppe terrestre valorisée de multiples façons. Le vénérable Paracelse recommandait déjà la Mumia Vera Aegyptica en tant que remède universel. Le commerce des cadavres embaumés a prospéré jusqu'aux années 1920. Sous forme de poudre pour gargarismes ou de complément alimentaire, les exportations faisaient un tabac et généraient des marges bénéficiaires élevées. Janine Kopp, journaliste et historienne, a obtenu son doctorat en 2012 à Lucerne avec une thèse intitulée "Hingerichtet und als Medikament verkauft» (Exécuté et vendu comme médicament). Au XVI et au XVII ${ }^{\mathrm{e}}$ siècles, les parties du corps les plus diverses étaient utilisées. Seuls les pharmaciens étaient légalement autorisés à pratiquer cette activité et transformaient par exemple la graisse des pendus en pommades ou leur boîte crânienne en poudres. Selon d'autres sources, les pharmaciens italiens fumaient de la viande musculaire séchée et suspendue dans leur cheminée.

Aujourd'hui, tout cela se fait de manière très stérile et réglementée par le biais d'entreprises telles que Tutogen Medical GmbH en Allemagne, une société au vaste portefeuille de solutions biologiques. La marchandise arrive cryogénisée de la clinique à la paillasse dans l'aile d'isolement. Les os humains sont transformés en chips spongieux et en chevilles, la peau de la cuisse, en filets pour les interventions chirurgicales sur la paroi abdominale. C'en est fini des glacières pleines de morceaux de corps ukrainiens depuis qu'un marchand de cadavres a livré un grand nombre d'os atteints par le cancer et le VIH avec des papiers falsifiés à la société mère américaine de Tutogen, entre autres. Il n'empêche que le commerce de tissus tels que les tendons, la peau, le cartilage, les os ou une partie de l'aorte en guise de substitut de valve cardiaque est en plein essor. Apparemment, les éléments du cadavre d'un seul être humain permettent de gagner jusqu'à 250000 dollars. Comme d'habitude, le quotidien Blick a une longueur d'avance. La cendre des incinérés est la nouvelle aubaine. Soleure et Zurich y recherchent de l'or, du platine et d'autres métaux précieux. Les recettes vont dans les caisses de l'Etat. eBay propose de longues listes d'implants usagés. Seuls les stimulateurs cardiaques doivent être retirés dans la chambre mortuaire avant la crémation: l'explosion de la pile au lithium peut endommager la paroi du four. L'électronique collectée est renvoyée au fabricant. Titane des implants dentaires, oxyde de zirconium, acier des tiges intramédullaires, des vis et plaques, chrome, molybdène, cobalt, palladium: tout est bon à prendre. Après la crémation à 900-1300 degrés au four électrique, la filtration et le tri magnétique commencent. L'or des dents et des bijoux, l'argent, le palladium et le platine pour les boutonspressions des implants, des appareils dentaires ou des bridges. Ce qui aidait à marcher sert maintenant pour la mastication et l'orthodontie. L'immortalité fonctionnelle ou la transmission du métal d'une génération à l'autre. Il existe plusieurs entreprises de recyclage. La situation juridique n'est pas claire. Pour Soleure, par exemple, les cendres n'ont pas de propriétaire et ne sont soumises à aucun droit de succession. Comme d'habitude, chaque canton a ses propres règles. Les cendres ne servent pas encore d'engrais dans les platesbandes des villes, ni à sécuriser les allées verglacées des cimetières, mais c'est tout juste.

Les cannibales sont plus appréciés que les détrousseurs de cadavres. Lorsque le défunt devient une marchandise du cycle de revalorisation, les conséquences sont profondes pour les vivants. Certes, chacun tire des bénéfices de ce cycle, mais quelque chose de fondamental se perd, avec l'aide de la médecine moderne.

\footnotetext{
Référence

- Janine Kopp, Menschenfleisch - Der menschliche Körper als Arz neimittel, 2014, à compte d'auteur.
} 